\title{
Sobre la representación del razonamiento basado en premisas*
}

\section{On the representation of premise-based reasoning}

\author{
ENRIC TRILLAS Y ALEJANDRO SOBRINO \\ Universidad de Santiago de Compostela
}

Recibido: 09/09/2020 Aceptado:18/11/2020

\begin{abstract}
RESUMEN
En este trabajo se expone una nueva forma de abordar el razonamiento basado en premisas, sosteniendo que razonar sobre algo consiste en conjeturar o refutar y que conjeturar sólo puede estribar en desarrollar lo que está oculto en las premisas, explicarlas o especular a partir de ellas. A continuación, se presenta el papel de los conjuntos borrosos para representar el significado impreciso característico del razonamiento ordinario, mostrando a la función de pertenencia como una aproximación de la medida en que un objeto verifica la propiedad denotada por el predicado vago o preciso. Finalmente, se indican algunas limitaciones del razonamiento de sentido común.
\end{abstract}

\section{PALABRAS CLAVE \\ CONJETURAS, HIPÓTESIS, ESPECULACIÓN, PREDICADOS VAGOS, CONJUNTOS BORROSOS}

\begin{abstract}
:
This paper deals with a new way of approaching the premise-based reasoning, suggesting that reasoning is conjecturing or refuting and that conjecturing is to figure out what is hidden in the premises, explaining them or speculating from them. Next, the role of fuzzy sets to represent the imprecise meaning characteristic of ordinary reasoning is addressed, showing the membership function as a kind of approximation to the extent to which an object satisfies the property

* Financiación a cargo del Ministerio de Economía y Competitividad y del European Regional Development Fund (ERDF/FEDER program), contratos TIN2014-56633-C3-1-R y TIN2017-84796-C2-1-R.
\end{abstract}

(C) Contrastes. Revista Internacional de Filosofía, vol. XXVI N² (2021), pp. 121-137. ISSN: 1136-4076

Departamento de Filosofía, Universidad de Málaga, Facultad de Filosofía y Letras

Campus de Teatinos, E-29071 Málaga (España) 
denoted by the vague or crisp spredicate. Finally, some limitations of the formalisms of the common-sense reasoning are addressed.

KEYWORDS

CONJECTURE, HYPOTHESIS, SPECULATION, VAGUE PREDICATES, FUZZY

SETS

\section{INTRODUCCIÓN: CONOCIMIENTO Y LÓGICA}

\section{1}

El PREMIO Nobel BRITÁNICo Sir Peter B. Medawar escribió: «Ningún proceso de razonamiento lógico permite ampliar el contenido informacional de los axiomas, premisas o enunciados observacionales de los que se parte» (Medavar, 1987). Se trata de una afirmación que merece detenerse en ella ya que contiene tres conceptos relevantes para analizar la relación entre conocimiento y lógica. El primero de esos conceptos es el de «proceso de razonamiento lógico» que sin duda substituye, al estar en conjunción con aquello de lo que se parte, a «proceso deductivo». El segundo, es el de «ampliar el contenido informacional» que, sin duda, se refiere a poder ir más allá de aquello de lo que se parte, de añadirle cosas nuevas. El tercero, es el de «axiomas, premisas o enunciados observacionales», que requiere distinguir entre, por lo menos, dos tipos de enunciados: los axiomas y los enunciados observacionales, como premisas de las que se parte. Ello conduce a diferenciar entre un tipo de búsqueda formal, como la de las matemáticas y una experimental, propia la de las ciencias naturales. Medawar indica que la deducción no sirve para hacer progresar el conocimiento hacia algo radicalmente nuevo, sino sólo para desarrollar, mostrar, lo que está implícito en las premisas o axiomas; que la creatividad requiere de procesos de razonamiento no deductivos, sino inductivos. Indica que la ciencia pretende buscar conocimiento nuevo a partir de datos observacionales adquiridos con la mayor seguridad posible por lo que se refiere a su validez. Todo ello requiere del lenguaje natural y del razonamiento ordinario, como también del razonamiento matemático previo a la prueba formal.

\section{2}

Conviene echar una mirada hacia el pasado remoto. Jenófanes de Colofón, entre los siglos VI y V A.C, ya se había dado cuenta de que cuanto sabemos sobre el mundo no es sino un tejido de conjeturas que, incluso si finalmente dejan de ser mantenidas, pueden permitir determinadas predicciones. Ese fue el caso 
del modelo ptolemaico geocéntrico, que permitía determinar bastante bien la posición de los planetas (a ojo desnudo) y aún pese a las complicaciones del «truco» de los epiciclos, y también del newtoniano para velocidades inferiores a la de la luz. Algo que, por sí mismo, hace que las conjeturas con alguna base observacional no deban ser vistas como despreciables. Con respecto al modelo de Ptolomeo, por ejemplo, hay que decir que en sí mismo no era propiamente especulativo, sino basado en las observaciones entonces posibles; lo que acabó siendo especulativo fue su mantenimiento por razones teológicas y apoyado en interpretaciones de la Biblia, arrogándose una justificación científica del que carecía, como ya entonces el uso observacional del telescopio ponía de relieve. Llegando a Aristóteles, en el siglo IV A.C, debe destacarse que es quien se preocupa de analizar cómo cabe inferir de modo seguro conclusiones a partir de premisas en sus modos silogísticos (Aristóteles, -345). Así, y pese a dejar de lado los razonamientos de tipo simplemente relacional, su estudio de los silogismos representa un gran paso adelante y se crea la lógica, el estudio del «logos» que acabará originando el de las consecuencias lógicas que, muchísimo más adelante y en el pasado siglo XX, Alfred Tarski formalizará con sus operadores de consecuencias (Tarski, 1936). «Logos» es un término de origen griego antiguo que se tradujo al latín por «ratio» y que admite traducciones como «afirmación», «discurso», «razonar», y que también podría significar un principio de orden y conocimiento.

Conviene distinguir, no obstante, entre las consecuencias lógicas de Tarski y las conclusiones obtenidas en el razonamiento ordinario. En las primeras, las consecuencias son seguras y, por tanto, pueden ser tomadas como nuevas premisas por más que no lleven a otra cosa que lo ya obtenido; en las segundas, sin embargo, no cabe hacer lo mismo, ya que pueden existir conclusiones contradictorias entre sí. ¿A qué es debida esta diferencia? Simplemente, a que Tarski formalizó la deducción formal, la típica de la prueba matemática, que no el razonamiento total de los matemáticos, el cual lo hacen como pueden, partiendo con frecuencia de analogías, especulando a partir de ellas, llegando a una hipótesis de lo que buscan, intentando refutarlas y, finalmente, probándolas a través de pasos mínimos apoyados en el Modus Ponens, que fue una de las importantes aportaciones de los lógicos medievales gracias, en parte, al éxito de las pruebas geométricas.

En el razonamiento ordinario, sin embargo, no cabe garantizar siempre que los pasos deductivos sean mínimos y, con ello, se abre la posibilidad de que haya agujeros de inferencia, saltos deductivos no apoyados en pasos elementales, que invaliden la prueba al abrir la posibilidad de otros caminos que puedan llevar eventualmente a otra parte. A este respecto, es ilustrativo lo sucedido tras la primera presentación de Andrew Wiles para demostrar el gran teorema de Fermat: hubo que esperar un tiempo hasta que un «salto mortal» 
que se le había escapado, y que fue detectado por otro matemático, fuese arreglado por el mismo Wiles y, sólo entonces, se aceptó la validez general del teorema de Fermat; sólo entonces ese teorema pasó a formar parte del «corpus» del conocimiento matemático (Singh, 2018). Un teorema que, hasta entonces, no era sino una conjetura que no había podido falsarse mediante contraejemplo alguno y que, por ello, se tenía la confianza en que se llegaría a probar deductivamente. Otro ejemplo, es el de las pruebas que durante el siglo XIX abundaron en algunos textos y según las cuales toda función continua es derivable; unas pruebas que incluían «saltos» como confundir la existencia de tangente en un punto con las pequeñas variaciones de la función alrededor de ese punto. Es la existencia de «saltos deductivos» lo que marca una notable diferencia entre la deducción formal y la de cada día. En la primera, el lenguaje es artificial y está reglamentado axiomáticamente, su textura es cerrada; en la segunda, el lenguaje es el natural y su textura es abierta. Por otra parte, el razonamiento ordinario también consta de «deducciones informales e inseguras», no es un razonamiento con red de seguridad, como lo es el deductivo formal.

\section{3}

Unos siglos antes de Tarski, en el XVII, el alemán Wilhem Gottfried Leibniz, influido por el Ars Magna de Ramon Llull, intentó establecer un cálculo con palabras sin lograrlo del todo; aunque no completó su Characteristica Universalis, creyó que llegaría un día en el que la gente en lugar de discutir se sentaría con papel y lápiz y podría resolver sus disputas argumentativas simplemente calculando conclusiones: «No discutamos, ¡calculemos!» (Leibniz, 1689). Sin embargo hubo que esperar hasta mediado el siglo XIX, cuando en su libro The Mathematical Analysis of Logic (Boole, 1847) el británico y matemático autodidacta George Boole hizo algo que marcaría por siempre el estudio de la lógica: construyó un modelo algebraico del razonamiento deductivo, un modelo puramente simbólico, introduciendo lo que luego se llamaría el álgebra de Boole, que ha llegado a ser el modelo típico tanto de la teoría de conjuntos, como del razonamiento deductivo clásico, que se hallan así imbricados. En el momento histórico en que surgía con fuerza y en Inglaterra el cálculo simbólico operacional, Boole, que había introducido el operador D para resolver por vía algebraica las ecuaciones diferenciales lineales, sustituyó frases lingüísticas por fórmulas y, operándolas con unas leyes parecidas a las de la aritmética, llegó a unas soluciones que, reinterpretadas lingüísticamente, eran las consecuencias. Por ejemplo, el silogismo:

Todos los hombres son mortales/Sócrates es un hombre: Sócrates es mortal, 
con la representación moderna del álgebra de Boole como un álgebra de conjuntos, queda recogido por la siguiente expresión formal:

$\mathrm{H} \subseteq \mathrm{M} \& \mathrm{~s} \in \mathrm{H}$, que implican la conclusión $\mathrm{s} \in \mathrm{M}$.

y su validez, usando la forma en la que Boole operaba, resulta del siguiente cálculo:

$$
\mathrm{H} \cdot \mathrm{M}=\mathrm{H} \& \mathrm{~s} \cdot \mathrm{H}=\mathrm{s} \rightarrow(\mathrm{s} \cdot \mathrm{H}) \cdot \mathrm{M}=\mathrm{s} \cdot(\mathrm{H} \cdot \mathrm{M})=\mathrm{s} \cdot \mathrm{H}=\mathrm{s} .
$$

Boole enseñó que los silogismos lingüísticos pueden representarse por sistemas de ecuaciones; fue un gran paso en la ruta del « ¿Calculemos!» de Leibniz, ya que no sólo enseñó eso, sino que la solución de esas ecuaciones lleva a la conclusión exacta de los silogismos. No obstante, el cálculo de Boole, luego perfeccionado por el alemán Ernst Schröder en su libro Vorlesungen über die Algebra der Logik, presupone muchísimas leyes que no son universalmente válidas en el lenguaje natural; así, la conmutatividad de la conjunción $(\mathrm{p} \cdot \mathrm{q}=\mathrm{q}$ - p) falla estrepitosamente en cuanto interviene el factor tiempo: «Fue juzgado y condenado» no es lo mismo que «Fue condenado y juzgado». Al ser casi permanente el juego temporal, no hay, por tanto, forma de capturar todos los matices del lenguaje natural con el álgebra de Boole; los conjuntos no bastan para ello y deben limitarse a sólo algunas de sus partes. En realidad, Boole quiso tomar parte a favor de Augustus de Morgan en una controversia acerca de un problema con los silogismos que, en Inglaterra, levantaba pasiones y que protagonizó con William Hamilton, un filósofo escocés casi olvidado. De hecho, la controversia, cerrada con el primer libro de Boole, era una lucha entre el razonamiento puramente lingüístico y el expresable por medio de las matemáticas, que ya empezaban a ser introducidas en mundos más allá de los de la mecánica y el calor. El mismo Boole, en 1856, lo amplió con multitud de aplicaciones en un segundo libro titulado, con cierta pretensión, An Investigation of the Laws of Thought (Boole, 1854).

Sin embargo, fue entre los siglos III y IV A.C., cuando Euclides de Alejandría (se tratase de una persona o un grupo de matemáticos) con sus conocidos Elementos (Euclides, -300), estableció definitivamente el ejemplo típico del razonamiento deductivo formal que, basado en postulados o axiomas, prueba los resultados o teoremas paso a paso con la ayuda del Modus Ponens. El crédito de este libro llevó, a lo largo de la Edad Media, a considerar al razonamiento deductivo como la única argumentación segura, al extremo de que el inglés Guillermo de Occam (siglos XIII y XIV), estableciese el principio metodológico conocido por la «navaja de Occam», según el cual no hay que introducir en las argumentaciones más que aquello estrictamente necesario para su prueba, algo equivalente al mínimo número de axiomas que 
son necesarios y suficientes para probar un teorema geométrico. Hubo que esperar hasta la mitad del pasado siglo XX para que Karl Menger añadiese su conocida adenda a la navaja de Occam: «ni menos de lo necesario para llegar deductivamente a algo significativo». Se trata de un precepto que tanto vale para un razonamiento deductivo como de otra clase; evitar introducir elementos impropios es algo que debe procurar quien intenta razonar correctamente.

\section{4}

En la mitad del pasado siglo XX, fue el norteamericano de adopción John von Neumann, nacido en Hungría como un súbdito del imperio austro-húngaro, quien llamó la atención sobre la imposibilidad del cálculo lógico clásico para representar enunciados que no son dicotómicos o bipolares; de respuesta «si» o «no» (von Neumann, 1951). Un guante que se lanzaba cuando la Cibernética, introducida por el norteamericano Norbert Wiener en un contexto más analógico que digital (Wiener, 1962), y donde tenían un papel más predominante las funciones que los símbolos, encaraban fenómenos tecnológicos no-lineales. Jóvenes ingenieros de electricidad y electrónica, como Lotfi A. Zadeh, se fijaron en esos problemas y propusieron modelos matemáticos y algorítmicos para, por ejemplo, la toma automática de decisiones en ámbitos que no tienen una caracterización precisa (Zadeh, 1965). A este respecto, Zadeh enunció el «principio de incompatibilidad», según el cual las descripciones precisas y significativas acerca del comportamiento de un sistema son inversamente proporcionales a su complejidad, de manera que relevancia y precisión son propiedades mutuamente excluyentes (Zadeh, 1973). Aparecía así la necesidad de encararse con las palabras que no significaban algo preciso, abundantes en el lenguaje y con las que verbalizamos la mayoría de nuestros problemas, no sólo los banales, sino también los importantes. Se trata de palabras cuyo significado no puede «definirse», sino más bien «describirse» por su uso en un contexto determinado; no cabe especificarlas en términos de condiciones necesarias y suficientes, de un «sí y sólo sí», como en matemáticas ocurre, por ejemplo, al definir un número primo; a lo sumo, se pueden dar reglas de uso que no son más que condiciones suficientes $\mathrm{y}$, sin embargo, con ellas vivimos, amamos, escribimos, trabajamos, o viajamos. Son palabras cruciales para entendernos y pelearnos, para sobrevivir; constituyen un continuo lingüístico imprescindible que, con poco gasto cognitivo, proporcionan la información necesaria para mantener el hilo conversacional. La imprecisión no debe confundirse con la incertidumbre (aunque la imprecisión suele arrojar alguna), a cargo de las medidas de probabilidad y posibilidad entre otras, y tampoco con la ambigüedad, que supone significados alternativos. 


\section{RAZONAR COMO CONJETURAR O REFUTAR.}

A continuación, propondremos que hacer para desanudar el «nudo gordiano» que constituyen tanto las representaciones matemáticas del lenguaje natural como del razonamiento de sentido común, y de lo cual la llamada lógica no-monótona no fue sino un minúsculo paso adelante (Trillas, 2015, 2018).

\section{II.1}

Uno de los conceptos que debe figurar entre los primeros a atender es el de rechazo o refutación de una conclusión. De abreviar el enunciado «Si p, entonces q» por $\mathrm{p}<\mathrm{q}$, y también abreviando «no q» por q', el enunciado «Si $\mathrm{p}$, entonces no-q» lo será por $\mathrm{p}<\mathrm{q}$ ', y el «No(Si p, entonces no-q)» lo será por $\mathrm{p}</$ q', que tanto puede concretarse en q' $<\mathrm{p}$ como en $\mathrm{p}$ NC q' (abreviando $\mathrm{NC}$, $\mathrm{p}$ no es comparable bajo la relación $<$ con q'). Cuando es $\mathrm{p}<\mathrm{q}$ ' se dirá que $\mathrm{p}$ y q son contradictorios; esto es, que de $\mathrm{p}$ sigue no-q. Debe notarse que en el cálculo lógico clásico $\mathrm{p}<\mathrm{q}$ ' (contradicción) es equivalente a $\mathrm{p} \cdot \mathrm{q}=0$ (incompatibilidad). Ir de la contradicción a la incompatibilidad exige: (i) la existencia de un enunciado, como es 0 , que sólo puede significar algo absurdo, (ii) la identificación de $<$ con el orden parcial reticular $\mathrm{p} \leq \mathrm{q} \Leftrightarrow \mathrm{p} \cdot \mathrm{q}=\mathrm{p}, \mathrm{y}$ (iii) la fortísima propiedad del reparto perfecto $\mathrm{p}=\mathrm{p} \cdot \mathrm{q}+\mathrm{p} \cdot \mathrm{q}$, de la cual sigue $\mathrm{p}=\mathrm{p} \cdot \mathrm{q} \Leftrightarrow \mathrm{p} \leq \mathrm{q}$ '. En cuanto al camino recíproco, de la contradicción a incompatibilidad, es: $\mathrm{p} \leq \mathrm{q}^{\prime} \Rightarrow \mathrm{p} \cdot \mathrm{q} \leq \mathrm{q}^{\prime} \cdot \mathrm{q} \Leftrightarrow \mathrm{p} \cdot \mathrm{q}=0$, que exige la monotonía del orden respecto de la conjunción y la radical propiedad q $\cdot \mathrm{q}^{\prime}=$ 0 .

Al cálculo clásico se le suponen una serie de propiedades que no se pueden suponer frecuentemente en el razonamiento ordinario (Trillas, GarcíaHonrado, 2013). Por ejemplo, la propiedad $p \cdot p^{\prime}=0$, que no hay nada entre $p$ y p', va en contra de la existencia de «infiltraciones informativas» entre p y p', así como implica que $\mathrm{p} \leq \mathrm{p}^{\prime}$ sólo pueda ser con $\mathrm{p}=0$, ya que $\mathrm{p} \leq \mathrm{p}^{\prime}=>\mathrm{p} \cdot \mathrm{p}=\mathrm{p}$ $\leq \mathrm{p}^{\prime} \cdot \mathrm{p}=0=>\mathrm{p}=0$, e. d., que sólo algo absurdo puede ser auto-contradictorio, cuando sabemos que con alguna frecuencia enunciamos conceptos autocontradictorios imprecisos, como son aquellos cuya función de pertenencia queda por debajo de la de su negación. La contradicción es, tanto de partida, como de llegada, el peor «pecado mortal» del razonamiento ordinario. No contradecirse es, seguramente, un postulado esencial que, como casi todos los de Euclides, todos estamos dispuestos a aceptar como evidente. El cálculo clásico, sin embargo, «agrupa» todas las auto-contradicciones en una única clase, la del elemento 0 y no permite distinguir entre ellas, al igual que no permite distinguir entre incompatibilidad y contradicción. El cálculo clásico se basa en un simbolismo que reúne un excesivo número de conceptos que 
no se agrupan necesariamente en el lenguaje ordinario; impone un lenguaje artificial demasiado simple.

\section{2}

Regresando a un «simbolismo menos restrictivo» en el cual $\mathrm{p}<\mathrm{q}$ signifique, simplemente, que hay una relación perceptiva de inferencia natural entre $\mathrm{p}$ y $\mathrm{q}$, para concluir $\mathrm{q}$ desde $\mathrm{p}$ se nos presentan dos posibilidades:

1) q es una conclusión-1 de $p$, si $p<q$,

2) q es una conclusión-2 de p, si p $</$ q',

de las cuales, la más general es la segunda, supuestas unas pocas propiedades de la relación $<$ y de la negación ', así como que p no sea auto-contradictorio. En efecto, si es $\mathrm{p}<\mathrm{q}$ y también fuese $\mathrm{p}<\mathrm{q}$ ', entonces, supuesta la propiedad $\left[a<b=>b^{\prime}<a^{\prime}\right]$, es q' $<p^{\prime} y$, supuesto también que $<$ es transitiva, de $p$ $<$ q' y q' < p', seguiría p < p', que es absurdo si no aceptamos puntos de partida auto-contradictorios. Toda conclusión-1 es una conclusión-2 con sólo suponer que la negación invierte la relación de inferencia natural $<$ y que ésta es transitiva. Diremos que una conclusión-2 es una conjetura de p y que una conclusión-1 es una consecuencia ordinaria de p. Alcanzar conjeturas es, con muy pocas propiedades, más general que alcanzar consecuencias; deducir es, como quería William Whewell, filósofo natural inglés de final del siglo XIX (Whewell, 1847), un caso particular de conjeturar.

Con ello, si $\mathrm{P}=\left\{\mathrm{p}_{1}, \ldots, \mathrm{p}_{\mathrm{n}}\right\}$ es un conjunto de enunciados o premisas que compactan lingüísticamente la información disponible sobre algo (por ejemplo, el comportamiento de un sistema dinámico, sea físico o virtual), y si p es un resumen de todas ellas, por ejemplo, su conjunción, una vez tomadas en una secuencia determinada y sugerida por el problema que sea (debe recordarse que no se supone que la conjunción sea ni conmutativa, ni asociativa), entonces, de existir los dos conjuntos:

$$
\operatorname{Conj}(\mathrm{P})=\left\{\mathrm{q} ; \mathrm{p}</ \mathrm{q}^{\prime}\right\}, \mathrm{y} \text { Cons }(\mathrm{P})=\{\mathrm{q} ; \mathrm{p}<\mathrm{q}],
$$

el segundo está contenido en el primero y, con solo suponer que siempre es $\mathrm{p}<$ p, Cons (P) no es vacío y tampoco lo es Conj (P). ¿Cómo clasificar Conj (P) Cons $(\mathrm{P})$ ? Es fácil, basta considerar los conjuntos $\operatorname{Hip}(\mathrm{P})=\{\mathrm{q} ; \mathrm{q}<\mathrm{p}\}$ y $\operatorname{Sp}(\mathrm{P})$ $=\{\mathrm{q} ; \mathrm{p} \mathrm{NC} \mathrm{q}\}$, de los que no es difícil probar que están en aquella diferencia de conjuntos, que son conjeturas y no son consecuencias. Por ejemplo, si es $\mathrm{q}<\mathrm{p}$ y fuese $\mathrm{p}<\mathrm{q}$, , seguiría $\mathrm{q}<\mathrm{q}$, , con lo que basta excluir de Hip (P) a los enunciados auto-contradictorios. Lo mismo cabe hacer, aunque sea un poco más difícil, con $\mathrm{Sp}(\mathrm{P})$, aunque nunca debemos llegar a conclusiones, 
sean del tipo que sean, auto-contradictorias. Por lo tanto, dado el conjunto de premisas $\mathrm{P}$, no hay más conjeturas que las consecuencias, las hipótesis y las especulaciones:

$$
\operatorname{Conj}(\mathrm{P})=\operatorname{Cons}(\mathrm{P}) \cup \operatorname{Hip}(\mathrm{P}) \cup \operatorname{Sp}(\mathrm{P}),
$$

reunión que es, evidentemente, una partición. Las conjeturas quedan, pues, perfectamente clasificadas en esos tres tipos y como el complementario de Conj $(\mathrm{P})$ es Conj $(\mathrm{P})^{\mathrm{c}}=\left\{\mathrm{q} ; \mathrm{p}<\mathrm{q}^{\prime}\right\}=\operatorname{Ref}(\mathrm{P})$, las refutaciones de $\mathrm{P}$, resulta, en definitiva, que los únicos enunciados relacionados con $\mathrm{P}$ por inferencia son:

$$
\operatorname{Conj}(\mathrm{P}) \cup \operatorname{Ref}(\mathrm{P})=\operatorname{Ref}(\mathrm{P}) \cup \operatorname{Cons}(\mathrm{P}) \cup \operatorname{Hip}(\mathrm{P}) \cup \operatorname{Sp}(\mathrm{P})
$$

A su vez, las especulaciones se clasifican en dos tipos disjuntos, ya que p NC q puede ir acompañado de q' $<$ p o de p NC q', quedando excluida únicamente la posibilidad de que sea $\mathrm{p}<\mathrm{q}$, que corresponde a una refutación. Así,

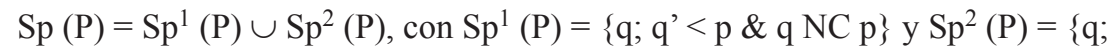
$\mathrm{p} \mathrm{NC} \mathrm{q} \mathrm{\&} \mathrm{p} \mathrm{NC} \mathrm{q' \} ,}$

siendo las primeras alcanzables por inferencia hacia atrás desde $\mathrm{p}$ hasta su negación q'y, como q' $<$ p implica p' $<\left(q^{\prime}\right)$ '), supuesto que la negación fuese «débil», es decir, verificase la propiedad (q')' < q, llegar a q. Las especulaciones de tipo uno, pueden, bajo determinadas condiciones, alcanzarse deductivamente, al igual que las hipótesis pueden alcanzarse deductivamente hacia atrás desde p. Sin embargo, las especulaciones de tipo dos, no pueden lograrse deductivamente ni hacia adelante ni hacia atrás, ya que no pueden existir caminos deductivos que desde p o p' las alcancen; las especulaciones de tipo dos, que se pueden denominar «creativas», son las conjeturas propiamente inductivas. Inducir es conseguir especulaciones creativas. Nótese que, por lo que respecta a las refutaciones, $\mathrm{p}<\mathrm{q}$ ' nos indica que q' es alcanzable deductivamente desde $\mathrm{p}$; con ello, q sería obtenible deductivamente supuesto que, o bien fuese q' $<$ q, o bien q < q', con lo cual y en el segundo caso, se trataría de una refutación auto-contradictoria que no aceptaríamos y quedando así las únicas posibilidades de que sea, o bien q' $<$ q, o bien q' NC q. La primera, en el caso clásico se reduce a $\mathrm{q}^{\prime} \leq \mathrm{q} \Leftrightarrow \mathrm{q}^{\prime}=0 \Leftrightarrow \mathrm{q}=1$ que indica un enunciado tautológico, uno comprendiendo todo lo suponible, la «verdad total» en términos clásicos; la segunda representa una refutación no obtenible por vía deductiva.

Por lo que respecta a qué sucede cuando aumenta el número de premisas, cuando aumenta la información previa, las consecuencias son monótonas; esto es, al aumentar el número de premisas, por lo menos no 
disminuye el de consecuencias; a más conocimiento, no cabe deducir menos, sólo cabe desarrollar lo que está oculto en las premisas. Las hipótesis y las especulaciones de tipo 1 son anti-monótonas; es decir, al aumentar las premisas, disminuyen las conclusiones. A más conocimiento caben menos explicaciones o especulaciones de tipo 1 y puede esperarse llegar a una definitiva. Las refutaciones también son monótonas, a más conocimiento, no cabe refutar menos. Las especulaciones de tipo 2, mantienen su carácter de inferencia «salvaje» ya que ni son monótonas ni anti-monótonas, carecen de una ley que nos permita prever si con más conocimiento caben más o menos de esas especulaciones; son, simplemente, no-monótonas (Trillas, 2017, 2018).

À la Popper, cabe resumir todo esto diciendo que razonar sobre algo consiste en, bien conjeturar, bien refutar, y que conjeturar sólo puede consistir en desarrollar lo que está oculto en las premisas, explicarlas o especular a partir de ellas. Queda, sin embargo, una pregunta importante: ¿Para qué especular? Es una pregunta de respuesta fácil:

Si $\mathrm{p}$ es el resumen de $\mathrm{P}$, y s es una especulación, supuesta la propiedad $\mathrm{p} \cdot \mathrm{s}<\mathrm{p}$, si $\mathrm{p} \cdot \mathrm{s}$ no es auto-contradictoria, es una hipótesis de P; es decir, explica P. Análogamente y supuesta la propiedad $p<p+s$, si $p+s$ no es auto-contradictoria, es una consecuencia de P. En conclusión, a través de una especulación se obtienen fácilmente tanto explicaciones, como consecuencias. Especulamos, por lo menos en parte, bien para desarrollar lo que conocemos, bien para explicarlo, e incluso si se especula sin pretender hacer ninguna de esas dos importantes cosas, especular es noble. Cabe, pues, especular para buscar más allá, para ampliar el contenido informacional de la información previa, para crear algo nuevo, para poder gritar a lo Arquímedes: «iEureka!» Nótese que la anterior fórmula $\mathrm{p} \cdot \mathrm{s}<\mathrm{p}$ indica que las hipótesis pueden obtenerse no sólo por vía deductiva sino, también inductiva.

\section{II.3}

Como final de este apartado, veamos como el simbolismo adoptado autoriza, con las poquísimas leyes aceptadas, a expresar de forma más general el principio de no-contradicción que, incluso, pasa a ser un teorema en contra de la afirmación de Aristóteles de que se trata de un principio simplemente evidente y no demostrable, y que enunció como la «imposibilidad» de los enunciados p · p' ( $\mathrm{y}$ y no $\mathrm{p}$ ). Supuesto $\mathrm{p} \cdot \mathrm{p}^{\prime}<\mathrm{p}$, y que $\mathrm{p} \cdot \mathrm{p}$ ' $<\mathrm{p}^{\prime}$, sigue $\mathrm{p}^{\prime}$ $<\left(p \cdot p^{\prime}\right)^{\prime} y$, por consiguiente $p \cdot p^{\prime}<\left(p \cdot p^{\prime}\right)^{\prime}$ : Los enunciados $p \cdot p^{\prime}$ son, siempre, auto-contradictorios. Nótese que, por tanto, en el cálculo clásico, ello se reduce a $\mathrm{p} \cdot \mathrm{p} '=0$, que es la forma usual en que se presenta el principio.

De forma análoga se llega al principio, ahora también teorema, del terceroexcluido que, en el cálculo clásico, se expresa por $\mathrm{p}+\mathrm{p}$ ' $=1$ y que en la forma «siempre es p o no p», se atribuye a Aristóteles, por más que no lo enunciase 
tan claramente como el principio anterior. De $p<p+p^{\prime}$ y p' $<$ p + p', sigue $(p$ $\left.+p^{\prime}\right)^{\prime}<p^{\prime} y$, por tanto, $\left(p+p^{\prime}\right)^{\prime}<p+p^{\prime} y$, finalmente, $\left(p+p^{\prime}\right)^{\prime}<\left(\left(p+p^{\prime}\right)^{\prime}\right)^{\prime}$ : Los enunciados de la forma ( $\left.\mathrm{p}+\mathrm{p})^{\prime}\right)$ ' son, siempre, auto-contradictorios. Por lo tanto, en el cálculo clásico es $\left(\mathrm{p}+\mathrm{p}^{\prime}\right)^{\prime}=0$, equivalente con $\mathrm{p}+\mathrm{p}^{\prime}=1$.

En conclusión, basta interpretar el «imposible» aristotélico como «autocontradictorio» para que los dos principios de no-contradicción y de tercero excluido (sobre los cuales muchos pensadores creen que los razonamientos se sostienen en un suelo firme) puedan demostrarse reduciendo su validez a unas, casi indudables, propiedades de $<$, ',$+\mathrm{y} \cdot$ Debe notarse que, de valer la ley de dualidad $(p \cdot q)$ ' $=$ p' + q', entonces ambos principios son equivalentes; sin embargo, en el lenguaje natural no es siempre el caso que la disyunción de las negaciones pueda ser equivalente a la negación de la conjunción.

Nótese que en estructuras como las álgebras de De Morgan, en las cuales no vale $\mathrm{x} \cdot \mathrm{x}^{\prime}=0\left(\Leftrightarrow \mathrm{x}+\mathrm{x}^{\prime}=1\right)$ para todo $\mathrm{x}$, se verifican ambos principios con sólo interpretar la relación de inferencia natural $<$ por el orden natural del retículo. Aun no tratándose todas ellas de retículos, los dos teoremas valen también en todas las álgebras de conjuntos borrosos, lo que viene a desmentir la tan común afirmación de que una característica de los mismos es la no validez de aquellos principios aristotélicos; al contrario, valen siempre sin más que considerar a $<$ como el orden puntual entre ellos e interpretar imposible como auto-contradictorio.

\section{LA REPRESENTACIÓN DEL SIGNIFICADO IMPRECISO}

\section{III.1}

La gran diferencia entre el lenguaje natural y los artificiales es que los segundos son casi puramente sintácticos, en tanto que el primero es semántico; no se puede aprender a hablar sin manejar adecuadamente y en cada contexto el significado de las palabras. En los lenguajes artificiales también hay contexto, pero viene dado por las reglas de construcción de los enunciados que en ellos se consideren válidos, aspirando a que lo sean en cualquier circunstancia. Esto es, sin embargo, algo escasamente razonable, como pretender que el lenguaje de las álgebras de Boole sea apropiado para expresar cualquier escenario lingüístico, cuando es sabido, por ejemplo, que la conjunción deja muchas veces de ser conmutativa, idempotente y distributiva respecto de la disyunción (axioma que, por ejemplo, ni siquiera es válido en el lenguaje de la física cuántica (Birkhoff \& von Neumann, 1936). Para abordar de forma efectiva cualquier representación del lenguaje natural más allá de lo preciso hay que ir hacia modelos matemáticos que puedan expresar en sus fórmulas los matices propios del significado de las palabras que integran las frases o enunciados (Trillas, 2017, 2018). Hay que saber cómo describir el significado de las 
palabras. En el caso de la imprecisión o vaguedad, los conjuntos borrosos han supuesto una importante contribución a esta tarea.

\section{2}

Sea $\mathrm{P}$ una palabra nombrando una propiedad que, eventualmente, muestren los elementos de un conjunto $\mathrm{X}$ o universo del discurso. $\mathrm{P}$ es un predicado en $\mathrm{X}$ (predica de sus elementos) y se trata, en primer lugar, de entender qué significan los enunciados elementales «X es $\mathrm{P}$ » que, conjuntamente, reflejan el significado de $\mathrm{P}$ en $\mathrm{X}$; se pasa, por tanto, del par (X, P), conjunto/predicado, al conjunto de enunciados lingüísticos $\{\mathrm{x}$ es $\mathrm{P} ; \mathrm{x} \in \mathrm{X}\}$, por más que los objetos de $\mathrm{X}$ tanto puedan ser físicos como virtuales o lingüísticos. Sólo existen dos posibilidades que puedan ser reconocidas de forma empírica; dados x e y de $\mathrm{X}$ :

1) Bien «x muestra menos que y» la propiedad nombrada por P, bien los enunciados «x es $\mathrm{P} » \mathrm{e}$ «y es $\mathrm{P} »$ no son comparables al respecto.

2) Bien «x muestra igual que y» la propiedad nombrada por P, bien ello no sucede; es decir, los enunciados «x es $\mathrm{P}$ » $\mathrm{e}$ «y es $\mathrm{P}$ » sólo transportan la información de que x e y satisfacen o no el predicado P; no hay más variaciones de esa satisfacción que «sí» o «no».

Designando por $<_{\mathrm{p}}$ la relación binaria «menos $\mathrm{P}$ que» $\mathrm{y}$ aceptando que $<_{\mathrm{P}}^{-1}$ designa la relación inversa «más $\mathrm{P}$ que» $\left(\mathrm{x}<_{\mathrm{P}}^{-1} \mathrm{y} \Leftrightarrow \mathrm{y}<_{\mathrm{P}} \mathrm{x}\right)$, cabe aceptar que la relación «igual que», designada por $=_{\mathrm{p}}$, corresponde a la intersección de $<_{\mathrm{P}} \mathrm{y}<_{\mathrm{P}}^{-1}$. Con ello, si las relaciones $<_{\mathrm{P}} \mathrm{y}=_{\mathrm{P}}$ son distintas, se dirá que $\mathrm{P}$ es impreciso en $\mathrm{X}$, en tanto que si coinciden $\left(<_{\mathrm{P}}==_{\mathrm{P}}\right)$ se dirá que $\mathrm{P}$ es preciso en $X$. En ambos casos, aceptaremos que el grafo $\left(X,<_{p}\right)$ representa el significado cualitativo de $\mathrm{P}$ en $\mathrm{X}$ : si $\mathrm{X}$ es preciso, ese grafo se reduce a $\left(\mathrm{X},=_{\mathrm{p}}\right)$. Debe notarse que no siempre es fácil conocer toda la relación $<_{\mathrm{p}}$, en tanto que de ordinario la ${ }_{\mathrm{p}}$ provendrá de una definición del estilo «x es $\mathrm{P} \Leftrightarrow$ tal y cual», con la cual es «x es igual $\mathrm{P}$ que $\mathrm{y} » \Leftrightarrow$ ambos $\mathrm{x}$ e y cumplen la definición. En el caso regular o impreciso, por tanto, se corre frecuentemente el riesgo de conocer únicamente una parte del significado cualitativo de $\mathrm{P}$ en $\mathrm{X}$, sin que ello quiera decir que en el caso preciso sea fácil determinar para cualquier x si verifica o no verifica la definición. Por ejemplo, en la recta real, el predicado $\mathrm{P}=$ trascendente es preciso, pero no es nada fácil comprobar el carácter trascendente de la mayoría de los números irracionales. Cuando la relación $<_{\mathrm{P}}$ es vacía, se dice que $\mathrm{P}$ carece de significado en $\mathrm{X}$ (meaningless, en inglés); cuando se desconoce del todo se dice que, actualmente, $\mathrm{P}$ es «inconmensurable» en $\mathrm{X}$; cuando se conoce sólo una parte, se dice que $\mathrm{P}$ es parcialmente medible en $\mathrm{X}$ y cuando se conoce toda ella, que es medible en $\mathrm{X}$. Está claro que si $\mathrm{P}$ es actualmente «inconmensurable», bien podrá llegar a carecer de significado, bien ser parcial o totalmente medible. 


\section{3}

Sea $\mathrm{P}$ medible en $\mathrm{X}$, con significado cualitativo $\left(\mathrm{X},<_{\mathrm{p}}\right)$. Se dice que $\mathrm{m}_{\mathrm{P}}$ : $X \rightarrow[0,1]$ es una medida del significado de $P$ en $X$, si verifica las tres leyes:

a) $\mathrm{x}<_{\mathrm{p}}$ y $\rightarrow \mathrm{m}_{\mathrm{p}}(\mathrm{x}) \leq \mathrm{m}_{\mathrm{p}}(\mathrm{y})$,

b) Si z es maximal en el grafo $\left(X,<_{\mathrm{p}}\right)$, es $\mathrm{m}_{\mathrm{p}}(\mathrm{z})=1$,

c) Si z es minimal, es $\mathrm{m}_{\mathrm{p}}(\mathrm{z})=0$.

De las tres, la primera es esencial y las otras dos sólo se verificarán de existir, respectivamente, maximales o minimales en el grafo (nótese que un elemento $\mathrm{x}$ de $\mathrm{X}$ puede tener medida 1 o 0 sin, por ello, ser maximal o minimal). Las tres leyes son condiciones necesarias pero no suficientes y, en particular, pueden existir $\mathrm{x}$ e y que, siendo $\mathrm{m}_{\mathrm{p}}(\mathrm{x}) \leq \mathrm{m}_{\mathrm{p}}(\mathrm{y})$, no sea $\mathrm{x}<_{\mathrm{p}} \mathrm{y}$. Es también importante notar que esas leyes describen a todas las posibles medidas, pero sin que ello baste para definir a una sola de ellas, como sucede, por ejemplo, con las propiedades básicas de las medidas de probabilidad, que no especifican a una sola de ellas.

Para especificar una medida debe añadirse información adicional, como sucede, también, con la probabilidad de obtener un resultado al lanzar un dado, caso en el que esa probabilidad es uniformemente 1/6 en la hipótesis de que el dado sea interna y externamente perfecto, la superficie de lanzamiento lisa e inelástica y su lanzamiento se haga correctamente. En el caso de que el dado sea «trucado» (por ejemplo, con un perdigón incrustado en la parte interior a la cara opuesta del seis), las probabilidades de los seis resultados posibles no serán $1 / 6 \mathrm{y}$, en particular, la de obtener seis será muy pequeña. En el caso lingüístico del predicado «probable», del que no es ni mucho menos inmediato que exista alguna medida $\mathrm{m}^{\text {probable }}$ que sea una probabilidad; una duda que proviene de que $\mathrm{m}^{\text {probable }}$ pueda satisfacer la ley «aditiva», ya que, en el caso impreciso, es difícil comprobar que «el crecimiento» de los enunciados «x es P» pueda representarse por conjuntos clásicos y en forma disjunta.

Ello ha comportado históricamente las discusiones entre «bayesianos» y «frecuentistas»; en todo caso y tras el trabajo de Andrei Kolmogorov en los años treinta, nadie duda hoy de que tanto la definición de probabilidad como la de probabilidad «a priori» dependen de información contextual y, con alguna frecuencia, son sensibles al contexto. La discusión no está cerrada y continúa, en cierta forma, con las probabilidades borrosas de Zadeh, del tipo prob(Juan es rico $)=$ alto - la probabilidad de que Juan sea rico es alta-, donde el enunciado es impreciso y por ello no es representable por un conjunto clásico, y el valor de la probabilidad no es un número real sino un conjunto borroso (Zadeh, 1984). Medir la incertidumbre lingüística es un tema realmente abierto. 


\section{III.4}

Una función de pertenencia al conjunto borroso de etiqueta lingüística $\mathrm{P}$ en $\mathrm{X}$, no es sino una medida del significado de $\mathrm{P}$ en $\mathrm{X} \mathrm{y}$, como es bien conocido, no hay una única función que defina al conjunto borroso, sino que son posibles muchas a partir de una misma serie de propiedades comunes. Por ejemplo, el predicado $\mathrm{P}=$ grande en el universo $\mathrm{X}=[0,10]$, cuyo significado cualitativo queda totalmente determinado en $[0,10]$ por $<_{\text {grande }}=\leq(\mathrm{el}$ orden total del intervalo $[0,10])$, tanto admite la medida $\mathrm{x} / 10$, como $\mathrm{x}^{2} / 100$, como aquella que vale 0 entre 0 y $5, y(x / 5)-1$ entre 5 y 10 , etc. Cada una de ellas se especifica a partir de información contextual sobre su forma; por ejemplo, para especificar la primera basta añadir a las tres propiedades genéricas: (a) es creciente; (b) vale 0 en $\mathrm{x}=0$; (c) vale 1 en $\mathrm{x}=10$, ya que sólo hay el minimal (mínimo) $\mathrm{x}=0$ y el maximal (máximo) $\mathrm{x}=10$. Obsérvese que la tercera medida añade muchos puntos con medida nula además del minimal 0 .

No obstante, y por lo que se dijo antes respecto a la dificultad de determinar toda la relación $<_{\mathrm{p}}$ (no siempre tan fácil de encontrar como en el ejemplo anterior), en general, lo máximo que se puede asegurar es que la función de pertenencia (fdp) es una aproximación a una medida. Es un problema abierto saber bajo qué condiciones cabe asegurar que, dado el significado cualitativo de $\mathrm{P}$ en $\mathrm{X}$, para cada fdp $\mu_{\mathrm{p}} \mathrm{y}$ cada $\varepsilon>0$, existe una medida $\mathrm{m}_{\mathrm{P}}$ tal que, para cada $\mathrm{x}$ de $\mathrm{X}$, sea $\left|\mu_{\mathrm{p}}(\mathrm{x})-\mathrm{m}_{\mathrm{p}}(\mathrm{x})\right| \leq \varepsilon$. Es decir, falta un teorema constructivo de cómo la fdp aproxima uniformemente a una medida, similar al que asegura que el control fuzzy se basa en un aproximador universal (Castro, 1995).

\section{CONCLUSIÓN}

\section{IV.1}

El lenguaje natural y el razonamiento de sentido común no están constreñidos por leyes estrictas rechazando determinadas construcciones sintácticas como sucede con los lenguajes artificiales de la lógica; el lenguaje natural va más allá de construcciones sintácticas erróneas que, incluso, permiten comprender enunciados mal construidos gramaticalmente (Aarts et al., 2004). Con frecuencia entendemos a los niños pequeños que balbuceando empiezan a hablar y que van aprendiendo por copia, error y rectificación. Gente que no ha ido nunca a la escuela y no saben leer ni escribir, se expresan oralmente de forma que no sólo se les entiende, sino que se puede discutir con ellos; realmente, dominan su idioma.

Los lenguajes artificiales son una parte muy pequeña del «lenguaje total» con el cual los pensamos y se diseñan, básicamente, para la deducción; tanto el cálculo lógico, como el cuántico, como los polivalentes o el borroso, no 
abarcan la totalidad de lo que es expresable en lenguaje natural. Ni siquiera tenemos criterios para saber cuándo los conectivos pueden expresarse funcionalmente; es, por ejemplo, el caso de la negación o el antónimo de un predicado que sólo sabemos representar, respectivamente, por una función de negación y por una simetría del universo, afectando la primera a los valores de la fdp y, la segunda, a los puntos del universo, y sin saber si ello es siempre correcto (Trillas, 2017). De lo que estamos seguros es de que no disponemos de expresiones universales de los conectivos como sucede en las lógicas clásica, cuántica y las polivalentes, que sólo aceptan conectivos distintos si se cambia de lógica; es decir, de cálculo. En el caso borroso, que permite representar parte de la imprecisión del lenguaje, ni siquiera sabemos si, en enunciados suficientemente largos, los conectivos de conjunción, de disyunción, de negación, de oposición y de condicionalidad, se mantienen constantes; por no referirnos a los cuantificadores y modificadores lingüísticos (Trillas, 2018). Además, seguimos con la metodología de intentar representar los enunciados por medio de sus componentes más elementales unidas por conectivos cuando es evidente que, con frecuencia y en el lenguaje natural, sólo capturamos el significado de tales componentes tras capturar el de todo el enunciado. Cargamos con unas tradiciones de análisis que, seguramente, hay que revisar.

\section{IV.2}

Todo ello cuando la conjunción de sensores y ordenadores y, en parte debido a la «fuerza bruta» que pueden desarrollar, están posibilitando que las máquinas empiecen a «hablar» con nosotros, aunque sea en contextos muy limitados. Los intentos de desatar el «nudo gordiano» de las representaciones matemáticas del lenguaje natural y del razonamiento de sentido común por medios lógicos no tiene un gran futuro; hay que proceder de otra forma que necesariamente ha de empezar por la observación más cuidadosa, seguida de modelos matemáticos y de experimentación controlada del manejo del lenguaje. Ello puede permitir aceptar o rechazar algunos modelos matemáticos, aunque sin ellos, y por simples que sean, no se puede «medir» y sin medir difícilmente cabe hacer una ciencia que pueda reproducir de manera mecánica el lenguaje y el razonamiento de sentido común.

\section{REFERENCIAS BIBLIOGRÁFICAS}

AARTS, B., D. DENISON, E. KEIZER, and G. POPOVA(eds.) 2004: Fuzzy Grammar. A Reader. Oxford University Press.

ARISTOTELES -345: Primeros Analíticos, Biblioteca Filosófica, Medina y Navarro, Volumen 7, Madrid, 1875. Traducción de Patricio de Azcárate. 
BIRKHOOF, G. \& J.VON NEUMANN 1936: «The Logic of Quantum Mechanics». The Annals of Mathematics, 2nd ser., Vol. 37, No. 4., pp. 823-843.

BOOLE, G., 1847: The Mathematical Analysis of Logic. Being an essay towards a calculus of deductive reasoning. MacMillan, Barclay \& MacMillan, London. Consultado en Googlebooks, 12/1/2020.

BOOLE, G., 1854: An Investigation of the Laws of Thought. The mathematical theories of logic and probabilities. Walton and Maberly. Cambridge. Consultado en Googlebooks, 12/1/2020.

CASTRO, J. L., 1995: «Fuzzy logic controllers are universal approximators», IEEE Trans on Systems, Man, and Cybernetics, Vol. 25, №. 4, pp. 629-635. https://doi. org/10.1109/21.370193

EUCLIDES, -300: Elementos. Universidad de Santiago de Compostela-Fundación BBVA, 2013.

KNEALE, W. \& KNEALE, M., 1962: The development of Logic. Oxford at the Clarendon Press.

LEIBNIZ. G. 1689: «Escritos lógico-matemáticos; Escritos sobre máquinas y ciencias físico-naturales». En, Obra completa, Javier Echeverría (ed.), Biblioteca de Grandes Pensadores. Editorial Gredos, 2011, pp. 277-506.

MEDAWAR, P., 1987: The Limits of Science, Oxford University Press, Oxford. Ver. cast. en Fondo de Cultura Económica, 1988.

POPPER, K.R. 1963: Conjectures and Refutations, Routledge \& Kegan Paul, London. RESCHER, N. 1969: Many-Valued Logic, McGraw-Hill, New York.

SCHRÖDER, E., 1890-1910: Algebra der Logik, Vols. I-III; reprint Chelsea 1966.

SINGH, S. 2018: «The Whole Story». Edited version of 2,000-word essay published in Prometheus magazine. https://simonsingh.net/books/fermats-last-theorem/ the-whole-story/ Consulta: 12/1/2018.

TARSKI, A. 1936: Introducción a la lógica y a las ciencias deductivas, Espasa-Calpe, 1985. V. orig. en Dover, 1936.

TRILLAS, E., GARCIA-HONRADO, I., 2013: «¿Hacia un replanteamiento del cálculo proposicional clásico?» Ágora, 12/1, pp. 7-25.

TRILLAS, E., 2015: Razonamiento, significado, incertidumbre y borrosidad. Publicaciones de la Universidad Pública de Navarra.

TRILLAS, E., 2017: On the Logos: A Naive View of Ordinary Reasoning and Fuzzy Logic. Springer, 2017.

TRILLAS, E., 2018: El desafio de la creatividad. Universidade de Santiago de Compostela editora.

VON NEUMANN, J 1951: «The General and Logical Theory of Automata», in Collected Works, (A. H. Taub ed.), vol. V, Pergamon Press, pp. 288-326

WHEWELL, W., 1847: The Philosophy of the Inductive Sciences, Founded Upon Their History, London: John W. Parker.

WIENER, N., 1962: Cybernetics, or control and communication in the animal and the machine, MIT Press, Cambridge, Massachusetts.

ZADEH, L. A., 1965: «Fuzzy sets», Information and control, vol. 8, pp. 338-353. 
ZADEH, L.A., 1973: «Outline of a New Approach to the Analysis of Complex Systems and Decision Processes», IEEE Trans. on Systems, Man, and Cybernetics, Vol. SMC-3, n 1 , pp. 28-44.

ZADEH, L. A., 1984: «Fuzzy probabilities», Information Processing \& Management, 20, No 3, pp. 363-372.

ENRIC Trillas es Profesor (jublado) de Ciencias de la Computación de la Inteligencia Artificial de la Universidad Politécnica de Madrid.

Línea de investigación

Razonamiento de sentido común, Lógica borrosa

Publicaciones recientes

Trillas y García-Honrado (2019):«Reflections on the Dialectic Synthesis». New Mathematics and Natural Computation, 1:316-328.

(2017): On the Logos. A Nä̈ve View on Ordinary Reasoning and Fuzzy Logic. Springer.

Correo electrónico: etrillasetrillas@gmail.com

Alejandro Sobrino es Profesor de Lógica y Filosofía de la ciencia de la Universidad de Santiago de Compostela.

Línea de investigación

Lenguaje, lógica y computación.

Publicaciones recientes

de Soto, Sobrino, Trillas y Alsina (2020): «Reflections on an old problem: That of preserving the logical forms and symmetry», Fuzzy Sets and Systems, vol 401, 2020, pp 150-162

(2019): «Cognitive Cities: An approach from citizens», en Designing Cognitive Cities.

R. Seising, M. E. Tabacchi \& Habenstein, A. (eds.), Springer, 2019, 135-148

Correo electrónico: alejandro.sobrino@usc.es 
